BMJ Open Diabetes Research \& Care

\title{
High or low glycemic index (GI) meals at dinner results in greater postprandial glycemia compared with breakfast: a randomized controlled trial
}

\author{
Sumanto Haldar (D) , ${ }^{1}$ Leonie Egli, ${ }^{2}$ Carlos Antonio De Castro, ${ }^{3}$ Shia Lyn Tay, \\ Melvin Xu Nian Koh, ${ }^{1}$ Christian Darimont, ${ }^{2}$ Katherine Mace, ${ }^{2}$ \\ Christiani Jeyakumar Henry ${ }^{1,4}$
}

To cite: Haldar S, Egli L, De Castro CA, et al. High or low glycemic index (Gl) meals at dinner results in greater postprandial glycemia compared with breakfast: a randomized controlled trial. BMJ Open Diab Res Care 2020;8:e001099. doi:10.1136/ bmjdrc-2019-001099

- Additional material is published online only. To view, please visit the journal online (http://dx.doi.org/10.1136/ bmjdrc-2019-001099).

Received 5 December 2019 Revised 3 March 2020 Accepted 9 March 2020

\section{Check for updates}

\section{(C) Author(s) (or their} employer(s)) 2020. Re-use permitted under CC BY-NC. No commercial re-use. See rights and permissions. Published by BMJ.

${ }^{1}$ Clinical Nutrition Research Centre, Singapore Institute for Clinical Sciences, Singapore ${ }^{2}$ Nestle Institute of Health Sciences, Lausanne, Switzerland

${ }^{3}$ Nestle Research Asia, Nestle Institute of Health Sciences, Singapore

${ }^{4}$ Department of Biochemistry, Yong L00 Lin School of Medicine, National University of Singapore, Singapore

\section{Correspondence to}

Professor Christiani Jeyakumar Henry;

jeya_henry@sics.a-star.edu.sg

\section{ABSTRACT}

Introduction While circadian control of glucose metabolism is well known, how glycemic index (Gl) of carbohydrate-rich meals interacts with time of consumption (breakfast or dinner) to influence postprandial (PP) glucose homeostasis is less well established. The objective of the study was to assess markers of PP glucose homeostasis following high or low Gl test meals (TM) consumed either at breakfast or at dinner and following consumption of the subsequent standardized meals (SSM). Research design and methods Randomized crossover trial in 34 healthy, Chinese, elderly volunteers (mean \pm SEM age, $56.8 \pm 0.83$ years), who completed 4 separate study sessions per-protocol, consisting of a highGl breakfast, low-Gl breakfast, high-Gl dinner and low-Gl dinner TM, followed by a SSM at the subsequent eating occasion. Blood samples were taken for 3 hours after each TM and SSM for glucose, insulin, glucagon, free fatty acids (FFA) and triglycerides (TG) measurements.

Results Consuming TM at dinner produced greater PP glycemia than breakfast both after TM and SSM (both $\mathrm{p}<0.0001$ ), irrespective of Gl. High-GI TM also produced greater PP glycemia than low-GI TM, both after TM and SSM (both $p<0.01$ ), irrespective of time of consumption. No interaction between Gl and time were found on PP glycemia, indicating parallel, but independent effects. Combined total areas under the curve of TM+SSM for PP glucose $(p<0.0001)$, PP TG $(p<0.0001)$ and PP FFA $(p<0.0001)$ were all greater when TM taken during dinner compared with breakfast.

Conclusions Carbohydrate-rich meals consumed at dinner leads to significantly worse PP glucose homeostasis than when consumed at breakfast, on top of the independent Gl effect of the meal. This may have implications to future type 2 diabetes risk. Moreover, future studies investigating Gl/glycemic load (GL) and disease risk associations should factor in timing of GL consumption as an additional variable.

Trial registration number NCT02927600.

\section{INTRODUCTION}

Postprandial glucose (PPG) homeostasis is an important determinant for the risk of chronic diseases, in particular type 2 diabetes and

\section{Significance of this study}

What is already known about this subject?

- Although diurnal variation in glucose metabolism via circadian control is well known, how this interacts with glycemic index/glycemic load (GI/GL) of meals to effect postprandial metabolism following the meals and following the subsequent meals is not established.

\section{What are the new findings?}

- This study has eloquently shown that carbohydraterich meals, particularly those high in $\mathrm{Gl}$, consumed during dinner, as compared with breakfast, gave rise to significantly adverse postprandial glucose homeostasis both after the meals and after the subsequent standardized meals.

How might these results change the focus of research or clinical practice?

- Given the worse glycemic control later during the day, it is advisable to focus attention on the carbohydrate quality and quantity of the evening meals, particularly in preventing future risk of type 2 diabetes and related cardiometabolic diseases.

- Future studies investigating $\mathrm{Gl} / \mathrm{GL}$ and disease risk associations should factor in timing of GL consumption as an additional variable.

cardiovascular diseases. ${ }^{1-3}$ Since the concept of glycemic index (GI) was propagated by Jenkins $e t a l,{ }^{4}$ low GI foods have been advocated to improve PPG and thereby manage and prevent type 2 diabetes, obesity and the risk of other cardiometabolic diseases. ${ }^{5-9}$ However, recent reviews have shown inconsistent effects of low GI foods in the prevention and management of chronic diseases. ${ }^{10-12}$ Part of these inconsistencies may be explained by diurnal variations in glucose homeostasis, which is often not considered in epidemiological studies. In fact, there has been a recent renaissance in research surrounding 
the circadian control of metabolism. ${ }^{13-15}$ The circadian rhythm is principally controlled centrally via the suprachiasmatic nucleus, as well as directly or indirectly through the peripheral clocks found in majority of the tissues to influence endocrine and metabolic functions including insulin secretion and insulin sensitivity. ${ }^{16}{ }^{17}$ While the central clock is mainly regulated by light-dark cycles, the peripheral clocks are much more influenced by behavioral patterns including fasting-feeding, sleep-awake cycles as well as by the dietary composition and the size of the meals. ${ }^{18}$ Therefore, eating meals at times mismatched to the internal clock, including having a nocturnal lifestyle have been shown to increase cardiometabolic disease burden. ${ }^{19}$ There are also suggestions of biological preferences for certain macronutrients at specific times of the day (eg, carbohydrate in the morning), as discussed in more detail elsewhere. ${ }^{20}$

Several studies have been undertaken which investigated how timing of meal intake interacts with nutrient profile to modulate metabolic homeostasis, ${ }^{18} 2122$ although majority of these evidence to date have come from controlled animal studies. More specifically, very few randomized controlled trials in humans, within the normal dietary context of consuming three mixed meals per day (at breakfast, lunch and dinner), have thoroughly explored how carbohydrate quality, particularly the GI/glycemic load (GL) of the meals interact with the timing of the meal intake to influence PPG homeostasis. Moreover, to the best of our knowledge, no study has investigated how the timing of the meal interacts with the GI of the same meal to influence the glycemic and insulinemic responses of the subsequent meal. Given that Asians have a greater predisposition to develop type 2 diabetes and prediabetes resulting from their distinctive metabolic phenotype, particularly in relation to carbohydrate metabolism, as compared with the Western Caucasian populations, ${ }^{23}{ }^{24}$ undertaking well-controlled trials in Asians to investigate interactions between meal timings and GI is imperative. We therefore investigated the effects of consuming high or low GI test meals (TM) either at breakfast or at dinner on various markers of PP glucose homeostasis immediately following TM as well as the PP period following the subsequent standardized meal (SSM).

\section{SUBJECTS AND METHODS}

\section{Study population and recruitment}

This study was undertaken in men and women of Chinese ethnic origin, 50-70 years of age and having a BMI between 18.5 and $24.9 \mathrm{~kg} / \mathrm{m}^{2}$. Exclusion criteria were: smoking, waist circumference $>90 \mathrm{~cm}$ (males) and $>85 \mathrm{~cm}$ (females), blood pressure $\geq 150 / 90 \mathrm{~mm} \mathrm{Hg}$, fasting glucose $\geq 7 \mathrm{mmol} / \mathrm{L}$, having alcohol consumption $>4$ units per day, having history of anemia, suffering from any medical conditions that may affect study results, being allergic/intolerant to any of the test foods or any common foods or ingredients, taking any prescribed medications or dietary supplements likely to interfere with study end points, being on any special or restrictive diet, for female volunteers: not reached menopause or being on hormone replacement therapy. The volunteers who took part in this study were recruited via advertisement in local newspaper, approaching potential volunteers from our center (Clinical Nutrition Research Centre (CNRC)) recruitment database, as well as through the word of mouth from August 2016 through to October 2017. Interested volunteers were invited to attend the CNRC for a screening and consent visit, following an overnight fast. Volunteers' suitability was determined using a Health and Lifestyle Questionnaire and through various anthropometric measurements. Participants' height (Seca 217, Seca GmbH, Germany), weight (Tanita BC-418, Tanita, Japan) and waist circumference were measured. Seated resting blood pressure was measured using an automated sphygmomanometer (Omron HEM907, Omron, Japan) and their fasting glucose using the HemoCue 201 (Radiometer, Denmark).

\section{Study design and dietary intervention}

This was a randomized, controlled, crossover, acute dietary intervention trial. The randomization was carried out using Medidata Balance (Medidata Solutions, USA) and the study team undertook recruitment and randomization. Each volunteer completed four separate intervention sessions for the study, in a random order, consisting of high-GI breakfast (Hi-Br), low-GI breakfast (Lo-Br), high-GI dinner (Hi-Di) and low-GI dinner (Lo-Di) as TM. All TM contained approximately $75 \mathrm{~g}$ available carbohydrates, made from either high GI rice (GI:92) or low GI rice (GI:55), flavored with a small amount of chicken seasoning and $20 \mathrm{~g}$ green leafy vegetables to ensure palatability. The GI values for the two rice were obtained by taking the measured values of the same types of rice used in two separate studies within our own research group. ${ }^{25}{ }^{26}$ Sessions were separated by a washout period of a minimum of 3 days. The meals immediately prior to each TM were standardized using standard meal (SM), meaning the same SM prior to each TM (high or low GI TM, taken either at breakfast or at dinner) was used in order to minimize any variations in baseline metabolite concentrations immediately prior to the TM, arising from any carryover effects from the volunteer's previous meal. For the two breakfast TM sessions, the volunteers attended the study center on the test day (day 1 ) at 7.30 am following an overnight fast lasting 13 hours, rested for approximately 1 hour following arrival and then had a cannula inserted around $8.30 \mathrm{am}$. After waiting for $15 \mathrm{~min}$ following cannula insertion, baseline blood sample (T0 hr) was obtained. At around $9.00 \mathrm{am}$, the volunteer consumed the TM breakfast (Hi-Br or Lo-Br), following which blood samples were obtained every $15 \mathrm{~min}$ for the first 1 hour and every $30 \mathrm{~min}$ for the subsequent 2 hours. Following this, another blood sample was drawn about 5 min before the SSM (standard lunch), which was consumed at 3.5 hours following the TM breakfast. 
Blood samples were again drawn every $15 \mathrm{~min}$ for the first 1 hour and every $30 \mathrm{~min}$ for the subsequent 2 hours following the SSM consumption, following which the cannula was removed and the volunteers were allowed to go home. Similarly, during the dinner TM sessions, the volunteers attended the study center at $5.00 \mathrm{pm}$ on test day (day 1), after previously eating a standardized lunch (SM, provided) at $12.30 \mathrm{pm}$ on the same day. They rested for approximately 1 hour following their arrival and then had a cannula inserted around $6.00 \mathrm{pm}$ on day 1 . After waiting $15 \mathrm{~min}$ following cannula insertion, baseline blood sample ( $\mathrm{T} 0 \mathrm{hr}$ ) was obtained. At around $6.30 \mathrm{pm}$, the volunteer consumed the test dinner (Hi-Di or Lo-Di) following which blood samples were obtained every $15 \mathrm{~min}$ for the first 1 hour and every $30 \mathrm{~min}$ for the subsequent 2 hours. Following this, the cannula was removed and the volunteers were allowed to go home. They were then asked to fast overnight (no food or drink other than water for the following 10 hours, approximately) and return to the study center the following morning (day 2) at $7.30 \mathrm{am}$, rested for approximately 1 hour following arrival and then had a cannula inserted around 8.30 am on day 2. After waiting for $15 \mathrm{~min}$ following cannula insertion, baseline blood sample ( $\mathrm{T} 0 \mathrm{hr}$ ) was obtained. At around 9.00 am (approximately 14.5 hours following their test dinner the evening before), volunteers consumed the SSM (standard breakfast) following which blood samples were drawn every $15 \mathrm{~min}$ for the first 1 hour and every $30 \mathrm{~min}$ for the subsequent 2 hours. The sequence and types of the TM and the standardized meals before and after TM (ie, SM and SSM, respectively) were identical irrespective of the intervention session, except for the time of consumption. The constituents of the test and standardized meals as well as their calorie and macronutrient compositions are shown in online supplementary table 1 . The schematic of study design is shown in online supplementary figure 1.

\section{Blood sample collection and analyses}

Blood samples were collected using an intravenous cannula by a qualified research nurse. Blood samples were either collected in serum vacutainer tubes (BD, USA) for the measurements of glucose, insulin and triglycerides (TG) or collected in $\mathrm{K}_{2}$ EDTA plasma vacutainer tubes (BD, USA) for the measurements of glucagon and free fatty acids (FFA), concentrations. The serum tubes were centrifuged within approximately 1 hour of collection after clot was formed. The plasma tubes were stored on ice immediately on collection and centrifuged within $30 \mathrm{~min}$ of collection. All blood tubes were centrifuged at $1500 \times g$ for $10 \mathrm{~min}$ at $4^{\circ} \mathrm{C}$. Serum and plasma samples were then aliquoted into individual cryotubes and immediately stored at $-80^{\circ} \mathrm{C}$ until analyses. Serum glucose, insulin and TG were measured using the standardized clinical chemistry method of the National University Hospital Referral Laboratories, Singapore, whereby glucose and TG were measured using AU 5800 clinical chemistry analyzer (Beckman Coulter, USA) and insulin was measured using UniCel DxI 800 Access Immunoassay System (Beckman Coulter, USA). Plasma FFA was measured by an in vitro enzymatic colorimetric method (Wako, Germany), while glucagon was measured by ELISAs (Mercodia, Sweden).

\section{Statistical analyses}

Sample size calculations and statistical analyses were implemented using R V.3.2.3 (R Foundation for Statistical Computing, Austria). Our previous data showed that a low GI breakfast significantly decreased interstitial glucose after breakfast and after lunch compared with a high GI breakfast. ${ }^{27}$ By assuming a lower effect size on glucose in an older population (75\% of the observed effect size in the reference study), a sample size of 35 volunteers was required $(1-\beta: 80 \%$; $\alpha=0.05)$.

Incremental areas under the curve (iAUCs) were calculated for each individual for each specific meal (TM and SSM). Incremental pertained to the correction of the baseline values immediately prior to the respective meals (ie, at $-5 \mathrm{~min}$ of the meal, respectively for TM and SSM). To do this, each respective baseline values were subtracted from the values observed after each meal at each given time point until the end of the 3-hour measurement period. The combined total AUC (tAUC) for each session was calculated by adding the total AUCs of both the TM and SSM within each session.

Linear mixed models were applied explaining the iAUCs or tAUCs combined of the meals according to the GI, the timing of intervention (using a $2 \times 2$ factorial design) as well as the interaction between the two factors: iAUC or tAUC $\sim \mathrm{GI}_{\text {high/low }} \times$ time of intervention breakfast/dinner . Assumptions were made for there being no carryover effect of one session to the next.

The above-mentioned model was used to make the following comparisons and obtain contrast estimates:

- High GI versus low GI (adjusting for time of intervention);

- Dinner versus breakfast (adjusting for GI);

- Within breakfast intervention: high GI versus low GI;

- Within dinner intervention: high GI versus low GI;

- Within high GI: dinner versus breakfast;

- Within low GI: dinner versus breakfast.

The same sets of analyses were performed on secondary outcomes such as blood insulin, TGs, FFAs and glucagon. Significance was set at $\mathrm{p}<0.05$. Adjustments for multiplicity were done using the False Discovery Rate method (also known as Hochberg and Benjamini method).

\section{RESULTS}

\section{Subjects and baseline characteristics}

A total of 62 volunteers were screened, 48 volunteers enrolled, 37 completed all 4 study sessions and 34 volunteers completed the study as per-protocol. The recruitment (Consolidated Standards of Reporting Trials) flow chart including the number of volunteers at the various stages of the study is shown in figure 1 . 


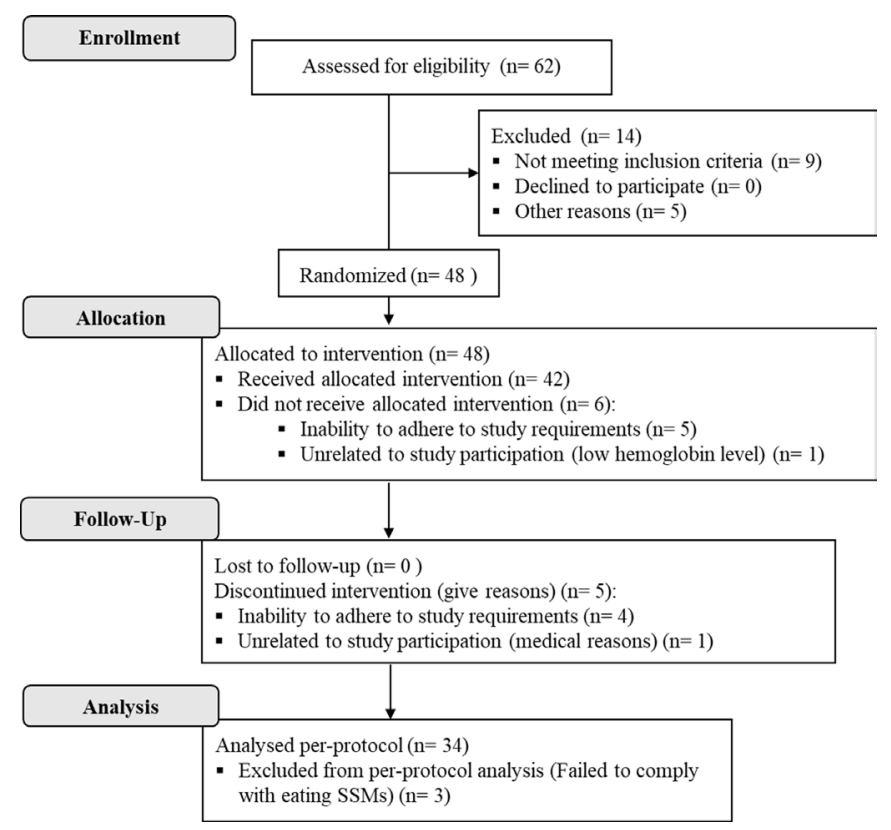

Figure 1 Consolidated Standards of Reporting Trials diagram. A total of 62 volunteers were screened, 48 volunteers enrolled, 37 completed all 4 study sessions and 34 volunteers completed the study as per-protocol. Of the total withdrawn participants, six withdrew from the study before the commencement of study intervention but after informed consent (ie, before session 1), four volunteers withdrew due to their inability to adhere to the study requirements (eg, study food consumption, not able to provide samples or undertake measurements) and one volunteer withdrew for medical reasons. SSM, subsequent standardized meal.

The detailed demographic characteristics of study participants is shown in table 1 . There were no differences in demographic characteristics between those enrolled into the study as compared with the per-protocol population, except for a larger proportion of male participants in the final per-protocol population. All female participants were postmenopausal.

The postprandial substrate and hormone concentrations following TM and SSM for up to 3-hour measurement periods after each meal during various intervention sessions are shown in figure 2. Baseline substrate and hormone concentrations before TM did not differ between high and low GI interventions, within breakfast or within dinner interventions ( $\mathrm{Hi}-\mathrm{Br}$ vs $\mathrm{Lo}-\mathrm{Br}$ and Hi-Di vs Lo-Di). However, baseline glucose, glucagon and TG concentrations immediately prior to TM were significantly greater in Hi-Di than Hi-Br TM sessions and TG concentration was greater in Lo-Di than Lo-Br TM sessions. Immediately prior to the SSM, baseline glucose and glucagon concentrations were greater following Lo-Br than Hi-Br TM sessions. Baseline TG concentration was greater while FFA was lower in Lo-Di than Hi-Di TM sessions. Baseline glucose and insulin concentrations were greater following breakfast TM sessions compared with dinner TM sessions, while that of glucagon, TG and FFA concentrations were lower following breakfast TM

\begin{tabular}{|c|c|c|c|}
\hline Variable & Level & $\begin{array}{l}\text { All subjects } \\
\text { enrolled }\end{array}$ & $\begin{array}{l}\text { Per-protocol } \\
\text { population }\end{array}$ \\
\hline $\mathrm{N}$ & & 48 & 34 \\
\hline BMI $\left(\mathrm{kg} / \mathrm{m}^{2}\right)$ & & $22.1 \pm 0.24$ & $22.3 \pm 0.29$ \\
\hline Height (cm) & & $164.1 \pm 1.11$ & $165.7 \pm 1.35$ \\
\hline Weight (kg) & & $59.7 \pm 1.24$ & $61.6 \pm 1.57$ \\
\hline $\begin{array}{l}\text { Waist circumference } \\
\text { (cm) }\end{array}$ & & $76.2 \pm 1.11$ & $77.8 \pm 1.39$ \\
\hline $\begin{array}{l}\text { Hip circumference } \\
(\mathrm{cm})\end{array}$ & & $94.0 \pm 0.77$ & $94.7 \pm 0.79$ \\
\hline SBP (mm Hg) & & $124.5 \pm 1.76$ & $122.6 \pm 2.09$ \\
\hline DBP (mm Hg) & & $78.3 \pm 1.11$ & $78.7 \pm 1.40$ \\
\hline $\begin{array}{l}\text { Fasting glucose } \\
(\mathrm{mmol} / \mathrm{L})\end{array}$ & & $4.6 \pm 0.07$ & $4.6 \pm 0.10$ \\
\hline Heart rate (bpm) & & $61.7 \pm 1.47$ & $62.2 \pm 2.00$ \\
\hline Age (years) & & $56.9 \pm 0.71$ & $56.8 \pm 0.83$ \\
\hline \multirow[t]{2}{*}{ Gender } & Female & 22 & 13 \\
\hline & Male & 26 & 21 \\
\hline Smoker & No & 48 & 34 \\
\hline \multirow[t]{2}{*}{ Alcohol consumption } & No & 40 & 28 \\
\hline & Yes & 8 & 6 \\
\hline
\end{tabular}

Data are expressed as mean \pm SEM; gender, smoker and alcohol consumption are expressed as number of subjects.

$\mathrm{BMI}$, body mass index; DBP, diastolic blood pressure; $\mathrm{N}$, number of subjects; SBP, systolic blood pressure.

sessions as compared with dinner sessions. The baseline substrate and hormone concentrations and the respective comparisons are shown in online supplementary table 2 .

\section{Postprandial metabolic responses (iAUCs) following TM and SSIM}

The iAUCs (which adjusts for baseline) after TM and SSM following various TM sessions are shown in table 2 . It should be noted that for $\mathrm{Br}$ TM sessions, the SSM were the subsequent lunch and for the Di TM sessions the SSM were the subsequent breakfast.

As expected, iAUCs of PPG $(\mathrm{p}<0.0001)$ and PPI $(\mathrm{p}<0.0001)$ following high GI TM was significantly greater than low GI TM, irrespective of meal time. More pertinently, iAUCs of PPG $(\mathrm{p}<0.0001)$ and PPI $(\mathrm{p}<0.05)$ following dinner TM was also greater than breakfast, irrespective of the GI content of the meals. While the interactions of GI:meal time were not significant for both PPG and PPI immediately after the TM (online supplementary table 3), the relative differences in iAUCs of PPG between dinner versus breakfast, irrespective of GI was greater than that between high versus low GI, irrespective of meal time. Similarly, iAUCs of PPG following SSM after high GI TM was significantly greater than the low GI TM sessions $(p<0.01)$, irrespective of meal time, although the iAUCs of PPI following SSM were not different between the high and low GI TM sessions. The iAUCs of both PPG $(p<0.0001)$ and PPI $(p<0.0001)$ following SSM after 
A
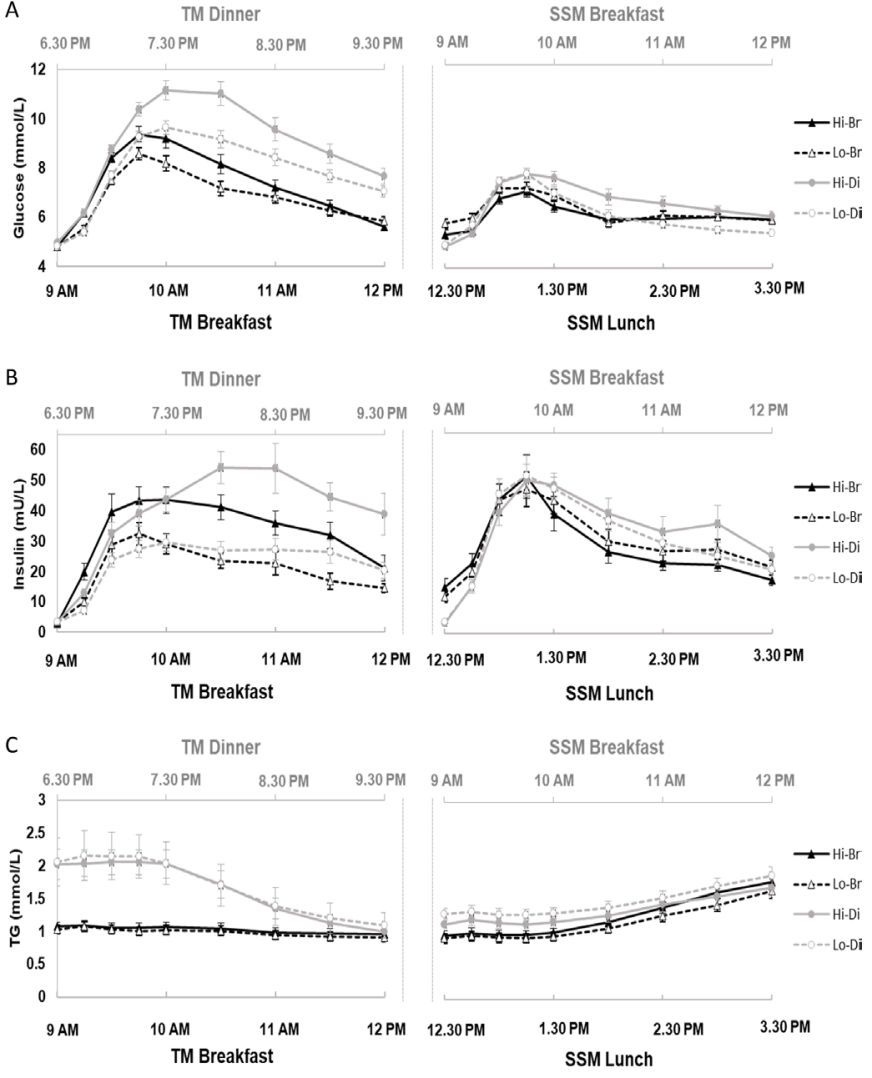

D
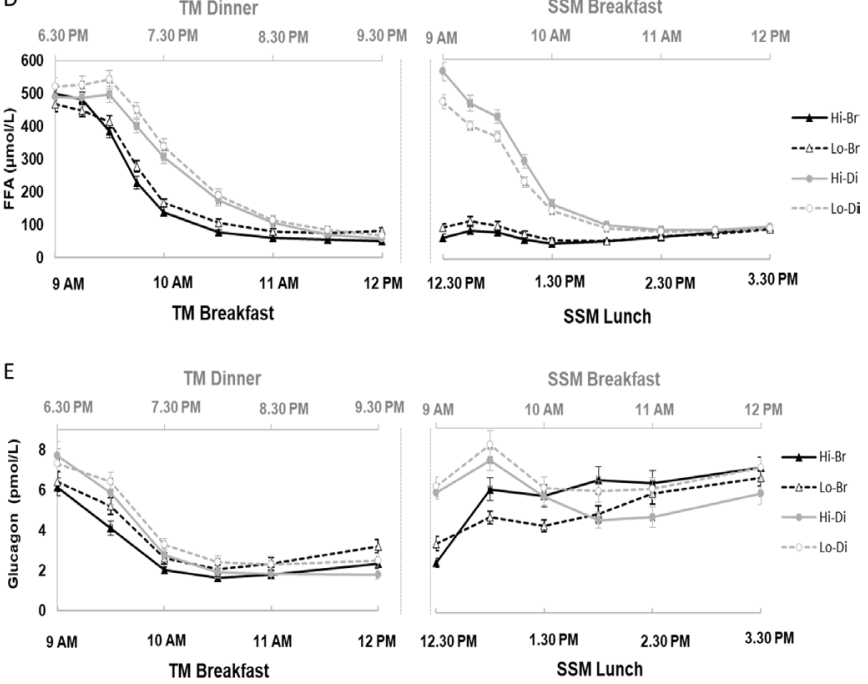

Figure 2 Postprandial substrate and hormone concentrations following test meals (TM) and subsequent standard meals (SSM) for up to 3-hour measurement periods after each meal. Mean \pm SEM concentrations of serum glucose $(A)$, serum insulin $(B)$, serum triglycerides $(T G)(C)$, plasma free fatty acids (FFA) (D) and plasma glucagon (E) after high-glycemic index breakfast (Hi-Br), low-glycemic index breakfast (Lo-Br), high-glycemic index dinner (Hi-Di), low-glycemic index dinner (Lo-Di) TM and SSM. For all variables, $n=34$ subjects, except for $F F A, n=29$.

dinner TM sessions were significantly greater than breakfast TM sessions, irrespective of the GI of previous TM. Indeed, there was a significant GI:meal time interaction $(p<0.05$; online supplementary table 3$)$ for the iAUC of
PPI following SSM, whereby for breakfast TM sessions, the high GI TM gave rise to a lower iAUCs of PPI after SSM than low GI TM session $(\mathrm{p}<0.05)$, although this association was not existent during dinner TM sessions.

For postprandial TG, while dinner had greater iAUCs than breakfast after TM, irrespective of GI $(p<0.001)$, no differences were found between high versus low GI, irrespective of meal time. However, for PP TG after SSM, while high GI TM sessions gave rise to greater iAUCs than low GI $(p<0.05)$, the iAUC of TG after SSM during dinner TM sessions were lower than the breakfast sessions $(p<0.0001)$. These observations though were most likely related to the differences in baseline concentrations of TG immediately prior to the SSM between various intervention sessions, possibly due to the differences between the dinner and breakfast sessions in the intermeal durations between TM and SSM. The postprandial suppression of FFA (inverse iAUC) following TM was significantly greater during breakfast than during dinner, irrespective of GI $(p<0.05)$, although there was no difference between high and low GI per se. Finally, postprandial glucagon suppression did not differ between treatments following TM. As for postprandial glucagon after SSM, there was significant interaction between GI:meal time $(\mathrm{p}<0.0001$; online supplementary table 3 ) and the detailed iAUC values and comparsions are shown in table 2. In general, the PP glucagon response following SSM (lunch) after breakfast interventions were significantly greater as compared with the SSM (breakfast) after dinner interventions, irrespective of the GI of the meals.

\section{Combined postprandial metabolic responses (tAUCs) of TM and SSIV}

The combined tAUCs of TM and SSM and the respective comparisons are shown in table 3. The combined tAUC of PP glycemia for high GI TM intervention session was significantly greater than the low GI. Similarly, the combined tAUC of PP glycemia for dinner was also greater than breakfast. There being a significant GI:meal time interaction $(p=0.001$; online supplementary table 4 ), while the high versus low GI difference was significant only during dinner interventions, the same was not true for the breakfast interventions. However, the dinner versus breakfast comparisons were significant, irrespective of the GI of the meals. The combined tAUC of PP insulin were also significantly greater for high versus low GI $(p<0.0001)$, as well as for dinner vs breakfast $(p<0.01)$ interventions.

The combined tAUC of PP TG was significantly greater during dinner intervention as compared with breakfast $(p<0.0001)$, although not between high versus low GI interventions. Similarly, irrespective of GI, there was a significant increase in combined tAUC of FFA during dinner TM as compared with breakfast TM session $(p<0.0001)$, although such effects were not found during high versus low GI comparisons. 
Table 2 Test meal (TM) and subsequent standard meal (SSM) incremental areas under the curve (iAUCs) of postprandial responses for various substrates and hormones

\begin{tabular}{|c|c|c|c|c|c|c|c|c|c|c|c|c|c|}
\hline \multirow[b]{2}{*}{ Variable } & \multirow[b]{2}{*}{ MT } & \multirow[b]{2}{*}{ GI } & \multirow[b]{2}{*}{$\mathbf{N}$} & \multicolumn{5}{|l|}{ TM } & \multicolumn{5}{|l|}{ SSM } \\
\hline & & & & Mean & SD & Comparison & Contrast & P value $^{\dagger}$ & Mean & SD & Comparison & Contrast & P value $^{\dagger}$ \\
\hline \multirow[t]{6}{*}{ Glucose } & $\mathrm{BR}$ & $\mathrm{Hi}$ & 34 & 481.99 & 192.50 & $\begin{array}{l}\text { Hi vs Lo } \\
\text { (overall) }\end{array}$ & 142.01 & $<0.0001$ & 140.70 & 155.36 & $\begin{array}{l}\text { Hi vs Lo } \\
\text { (overall) }\end{array}$ & 70.96 & $<0.01$ \\
\hline & & Lo & 34 & 378.64 & 158.75 & Di vs $\mathrm{Br}$ (overall) & 245.75 & $<0.0001$ & 93.83 & 156.48 & Di vs Br (overall) & 155.78 & $<0.0001$ \\
\hline & DI & $\mathrm{Hi}$ & 34 & 766.40 & 320.61 & Br: Hi vs Lo & 103.35 & $<0.05$ & 320.57 & 151.72 & Br: Hi vs Lo & 46.87 & 0.1836 \\
\hline & & Lo & 34 & 585.74 & 215.50 & Di: Hi vs Lo & 180.66 & $<0.0001$ & 225.52 & 108.36 & Di: Hi vs Lo & 95.05 & $<0.01$ \\
\hline & & & & & & Hi: Di vs Br & 284.40 & $<0.0001$ & & & Hi: Di vs Br & 179.88 & $<0.0001$ \\
\hline & & & & & & Lo: Di vs Br & 207.10 & $<0.0001$ & & & Lo: Di vs Br & 131.69 & $<0.001$ \\
\hline \multirow[t]{6}{*}{ Insulin } & $\mathrm{BR}$ & $\mathrm{Hi}$ & 34 & 5643.62 & 3093.41 & $\begin{array}{l}\text { Hi vs Lo } \\
\text { (overall) }\end{array}$ & 2729.25 & $<0.0001$ & 2447.71 & 1651.66 & $\begin{array}{l}\text { Hi vs Lo } \\
\text { (overall) }\end{array}$ & -232.46 & 0.4477 \\
\hline & & Lo & 34 & 3269.08 & 2073.61 & Di vs Br (overall) & 865.96 & $<0.05$ & 3454.72 & 2338.81 & Di vs Br (overall) & 2462.41 & $<0.0001$ \\
\hline & $\mathrm{DI}$ & $\mathrm{Hi}$ & 34 & 6864.29 & 3714.81 & Br: Hi vs Lo & 2374.54 & $<0.0001$ & 5684.67 & 3420.26 & Br: Hi vs Lo* & -1007.01 & $<0.05$ \\
\hline & & Lo & 34 & 3692.37 & 2039.35 & Di: Hi vs Lo & 3083.97 & $<0.0001$ & 5141.35 & 2288.56 & Di: Hi vs Lo* & 542.09 & 0.2398 \\
\hline & & & & & & Hi: Di vs Br & 1220.67 & $<0.05$ & & & Hi: Di vs Br & 3236.96 & $<0.0001$ \\
\hline & & & & & & Lo: Di vs Br & 511.24 & 0.3481 & & & Lo: Di vs Br* & 1687.85 & $<0.001$ \\
\hline \multirow[t]{6}{*}{ Triglycerides } & $\mathrm{BR}$ & $\mathrm{Hi}$ & 34 & 3.98 & 7.93 & $\begin{array}{l}\text { Hi vs Lo } \\
\text { (overall) }\end{array}$ & 0.77 & 0.7772 & 53.62 & 27.28 & $\begin{array}{l}\text { Hi vs Lo } \\
\text { (overall) }\end{array}$ & 7.21 & $<0.05$ \\
\hline & & Lo & 34 & 2.58 & 3.60 & Di vs $\mathrm{Br}$ (overall) & 9.66 & $<0.001$ & 42.93 & 21.43 & Di vs Br (overall) & -13.42 & $<0.001$ \\
\hline & DI & $\mathrm{Hi}$ & 34 & 13.02 & 18.47 & Br: Hi vs Lo & 1.40 & 0.7465 & 36.72 & 36.57 & Br: Hi vs Lo & 10.69 & $<0.05$ \\
\hline & & Lo & 34 & 12.87 & 18.88 & Di: Hi vs Lo & 0.15 & 0.9645 & 32.99 & 30.08 & Di: Hi vs Lo & 3.73 & 0.4473 \\
\hline & & & & & & Hi: Di vs Br & 9.04 & $<0.05$ & & & Hi: Di vs Br & -16.90 & $<0.01$ \\
\hline & & & & & & Lo: $\mathrm{Di}$ vs $\mathrm{Br}$ & 10.29 & $<0.01$ & & & Lo: $\mathrm{Di}$ vs $\mathrm{Br}$ & -9.94 & $<0.05$ \\
\hline \multirow[t]{6}{*}{$\begin{array}{l}\text { Free fatty } \\
\text { acids }\end{array}$} & $\mathrm{BR}$ & $\mathrm{Hi}$ & 29 & -59504.89 & 26273.50 & $\begin{array}{l}\text { Hi vs Lo } \\
\text { (overall) }\end{array}$ & -3703.86 & 0.3504 & 496.98 & 7870.64 & $\begin{array}{l}\text { Hi vs Lo } \\
\text { (overall) }\end{array}$ & -3967.37 & 0.1552 \\
\hline & & Lo & 30 & -50405.09 & 18057.99 & Di vs $\mathrm{Br}$ (overall) & 8766.81 & $<0.05$ & -3446.39 & 7360.76 & $\mathrm{Di}$ vs $\mathrm{Br}$ (overall) & -56096.50 & $<0.0001$ \\
\hline & $\mathrm{DI}$ & $\mathrm{Hi}$ & 29 & -45272.73 & 22182.40 & Br: Hi vs Lo & -8909.32 & 0.1022 & -63524.99 & 24943.72 & Br: Hi vs Lo* & 4231.63 & 0.2678 \\
\hline & & Lo & 29 & -46774.32 & 26604.49 & Di: Hi vs Lo & 1501.59 & 0.7772 & -51358.61 & 17701.28 & Di: Hi vs Lo* & -12166.38 & $<0.01$ \\
\hline & & & & & & $\mathrm{Hi}$ : Di vs Br & 13972.27 & $<0.05$ & & & Hi: Di vs $B r^{*}$ & -64295.50 & $<0.0001$ \\
\hline & & & & & & Lo: Di vs Br & 3561.36 & 0.5193 & & & Lo: Di vs Br & -47897.49 & $<0.0001$ \\
\hline \multirow[t]{6}{*}{ Glucagon } & $\mathrm{BR}$ & $\mathrm{Hi}$ & 34 & -637.76 & 354.44 & $\begin{array}{l}\text { Hi vs Lo } \\
\text { (overall) }\end{array}$ & -118.79 & 0.1233 & 647.97 & 374.81 & $\begin{array}{l}\text { Hi vs Lo } \\
\text { (overall) }\end{array}$ & 111.58 & $<0.05$ \\
\hline & & Lo & 34 & -564.90 & 333.75 & Di vs $\mathrm{Br}$ (overall) & -126.52 & 0.1022 & 317.80 & 293.40 & Di vs Br (overall) & -435.34 & $<0.0001$ \\
\hline & $\mathrm{DI}$ & $\mathrm{Hi}$ & 34 & -820.98 & 502.17 & Br: Hi vs Lo & -93.00 & 0.3889 & -48.14 & 395.24 & Br: Hi vs Lo* & 347.53 & $<0.0001$ \\
\hline & & Lo & 34 & -671.83 & 589.98 & Di: Hi vs Lo & -144.58 & 0.1878 & 77.36 & 447.54 & Di: Hi vs Lo* & -124.36 & 0.1195 \\
\hline & & & & & & Hi: Di vs Br & -152.31 & 0.1653 & & & Hi: Di vs Br* & -671.28 & $<0.0001$ \\
\hline & & & & & & Lo: Di vs $\mathrm{Br}$ & -100.73 & 0.3619 & & & Lo: Di vs $B r^{*}$ & -199.39 & $<0.05$ \\
\hline
\end{tabular}

Linear mixed models were used to compare the iAUCs following TM according to the Gl and MT in a $2 \times 2$ analyses. Significance was set at $p<0.05$. 'Hi vs Lo overall' - when dinner and breakfast combined. 'Di vs Br overall' - when high and low Gl combined. Bold values are statistically significant.

*Adjustments for multiplicity was done using the False Discovery Rate method.

tOnly when GI:MT interaction significant, subgroup analyses are shown in italics.

$\mathrm{BR}$, breakfast; $\mathrm{DI}$, dinner; GI, glycemic index; Hi, high Gl; Lo, low GI; MT, meal time; N, number.

\section{DISUSSION}

The study showed that the time of consumption (in this case breakfast vs dinner) of a carbohydrate-rich meal had significant effects on various markers of PP glucose homeostasis in the PP period immediately after the meal itself and after the SSM, on top of the independent effects of GI. While the differences between high and low GI meals on PP glycemia and insulinemia were expected, the differences in PPG and PPI between dinner and breakfast, irrespective of their GI content are likely to be physiologically relevant, with potentially significant public health implications. This observation is particularly important since majority of the epidemiological studies investigating associations between GI/GL of the diet and disease risk generally ignore considerations on assessing the timing of the consumption in addition to calculating the mean daily GI/GL contents of the foods. For example, an individual habitually consuming proportionately greater GL (amount of available carbohydrate $\times$ GI) during dinner may have an overall worse glycemic status than someone else who habitually consumes greater GL for breakfast, even though their mean daily GL may be similar. This observation may partly explain some of the heterogeneity and inconsistencies in the GI-disease associations between various studies. ${ }^{10-12}$ 
Table 3 Combined total areas under the curves (tAUCs) of test meals (TM) and subsequent standard meal (SSM) postprandial responses for various substrates and hormones

\begin{tabular}{|c|c|c|c|c|c|c|c|c|}
\hline Variable & Meal time & GI & $\mathbf{N}$ & Mean & SD & Comparison & Contrast & $\mathbf{P}$ value† \\
\hline \multirow[t]{6}{*}{ Glucose } & $\mathrm{BR}$ & $\mathrm{Hi}$ & 34 & 2493.32 & 334.44 & Hi vs Lo (overall) & 181.11 & $<0.0001$ \\
\hline & & Lo & 34 & 2423.70 & 328.43 & Di vs $\mathrm{Br}$ (overall) & 296.81 & $<0.0001$ \\
\hline & $\mathrm{DI}$ & $\mathrm{Hi}$ & 34 & 2901.62 & 442.64 & Br: Hi vs Lo* & 69.63 & 0.1906 \\
\hline & & Lo & 34 & 2609.02 & 306.35 & Di: Hi vs Lo* & 292.60 & $<0.0001$ \\
\hline & & & & & & Hi: Di vs $B r^{*}$ & 408.29 & $<0.0001$ \\
\hline & & & & & & Lo: Di vs Br & 185.32 & $<0.001$ \\
\hline \multirow[t]{6}{*}{ Insulin } & $\mathrm{BR}$ & $\mathrm{Hi}$ & 34 & 11425.73 & 6261.91 & Hi vs Lo (overall) & 2686.65 & $<0.0001$ \\
\hline & & Lo & 34 & 9516.74 & 4963.53 & Di vs $\mathrm{Br}$ (overall) & 1572.55 & $<0.01$ \\
\hline & $\mathrm{DI}$ & $\mathrm{Hi}$ & 34 & 13775.94 & 6962.72 & Br: Hi vs Lo & 1908.99 & $<0.01$ \\
\hline & & Lo & 34 & 10126.83 & 4127.53 & Di: Hi vs Lo & 3464.31 & $<0.0001$ \\
\hline & & & & & & $\mathrm{Hi}$ : Di vs $\mathrm{Br}$ & 2350.21 & $<0.01$ \\
\hline & & & & & & Lo: Di vs $\mathrm{Br}$ & 794.89 & 0.3125 \\
\hline \multirow[t]{6}{*}{ Triglycerides } & $\mathrm{BR}$ & $\mathrm{Hi}$ & 34 & 421.39 & 156.72 & Hi vs Lo (overall) & -3.30 & 0.9097 \\
\hline & & Lo & 34 & 395.60 & 155.64 & Di vs $\mathrm{Br}$ (overall) & 158.25 & $<0.0001$ \\
\hline & $\mathrm{DI}$ & $\mathrm{Hi}$ & 34 & 550.54 & 278.76 & Br: Hi vs Lo & 25.79 & 0.5690 \\
\hline & & Lo & 34 & 582.93 & 404.90 & Di: Hi vs Lo & -32.39 & 0.4981 \\
\hline & & & & & & $\mathrm{Hi}$ : Di vs $\mathrm{Br}$ & 129.16 & $<0.01$ \\
\hline & & & & & & Lo: Di vs Br & 187.33 & $<0.0001$ \\
\hline \multirow[t]{6}{*}{ Free fatty acids } & $\mathrm{BR}$ & $\mathrm{Hi}$ & 29 & 44669.53 & 14708.28 & Hi vs Lo (overall) & -2131.99 & 0.4862 \\
\hline & & Lo & 30 & 50423.81 & 17067.00 & Di vs $\mathrm{Br}$ (overall) & 36217.11 & $<0.0001$ \\
\hline & $\mathrm{DI}$ & $\mathrm{Hi}$ & 29 & 84738.51 & 17216.24 & Br: Hi vs Lo & -5761.22 & 0.1680 \\
\hline & & Lo & 29 & 83241.27 & 18907.22 & Di: Hi vs Lo & 1497.24 & 0.7035 \\
\hline & & & & & & $\mathrm{Hi}: \mathrm{Di}$ vs $\mathrm{Br}$ & 39846.33 & $<0.0001$ \\
\hline & & & & & & Lo: Di vs $\mathrm{Br}$ & 32587.88 & $<0.0001$ \\
\hline \multirow[t]{6}{*}{ Glucagon } & $\mathrm{BR}$ & $\mathrm{Hi}$ & 34 & 1601.86 & 560.85 & Hi vs Lo (overall) & -81.57 & 0.1906 \\
\hline & & Lo & 34 & 1563.72 & 570.10 & Di vs $\mathrm{Br}$ (overall) & 207.56 & $<0.001$ \\
\hline & $\mathrm{DI}$ & $\mathrm{Hi}$ & 34 & 1638.99 & 659.15 & Br: Hi vs Lo* & 55.67 & 0.5241 \\
\hline & & Lo & 34 & 1885.22 & 766.10 & Di: Hi vs Lo* & -218.82 & $<0.001$ \\
\hline & & & & & & Hi: Di vs $B r^{*}$ & 70.31 & 0.4604 \\
\hline & & & & & & Lo: Di vs $B r^{*}$ & 344.80 & $<0.0001$ \\
\hline
\end{tabular}

Linear mixed models were used to compare the combined tAUCs following TM+SSM according to the GI and MT in a $2 \times 2$ analyses.

Significance was set at $p<0.05$.

'Hi vs Lo overall' - when dinner and breakfast combined. 'Di vs Br overall'-when high and low Gl combined.

Bold values are statistically significant.

*Only when GI:MT interaction significant, subgroup analyses are shown in italics.

†Adjustments for multiplicity was done using the False Discovery Rate method.

BR, breakfast; DI, dinner; GI, glycemic index; Hi, high Gl; Lo, low Gl; MT, meal time; N, number.

In support of our findings, a recent study comparing the response with a low GI dinner (taken at $8.00 \mathrm{pm}$ ) and breakfast (taken at $8.00 \mathrm{am}$ ) in a young non-diabetic population also found that the glucose iAUC at dinner was significantly greater than breakfast. ${ }^{28}$ In their study, Leung et al kept the duration of the prior fasting period identical for both conditions, hence excluding the potential confounding role of the intermeal duration factor. In our study however, while the duration of the fasting periods before TM as well as before SSM were indeed variable between dinner and breakfast interventions, these meal timings and intermeal durations reflected a normal dietary pattern of 3 meals per day, that is, breakfast in the morning, lunch in the early afternoon and dinner in the evening. Similar to our findings, another study found that the timing of the day had greater effects on PPG than the difference between high and low GI meals in a young non-diabetic population. ${ }^{29}$ One of the differences between our study and the two previously cited studies were the population recruited. Indeed, we 
included an older cohort in whom glucose homeostasis was likely to be more compromised ${ }^{30}$ and who could benefit more in terms of cardiometabolic disease prevention. The strength of our study design as compared with these earlier studies was the use of a larger sample size (34 vs 9 and 10, respectively). Moreover, we studied participants of Chinese origin, known to be at a greater risk of developing type 2 diabetes than Caucasians, ${ }^{24} 31$ and our study had been one of the most comprehensive studies to investigate simultaneously the influences of timing and GI variations of meals on PPG responses in Asians. Overall, the results of our study and previous findings corroborate that in non-diabetic individuals, glucose metabolism is significantly worse in the evenings in comparison to the mornings, irrespective of the GI content of the meal.

While it has been long known that the composition (including GI/GL) of the previous meal affects the glycemic responses of the subsequent meal, ${ }^{32-34}$ no previous study has investigated the effect of meal time (breakfast vs dinner) and GI variations on the subsequent meal effect concurrently. We found that both differences in the timing of previous meal (TM) intake as well as the variations in GI independently and simultaneously affected the PPG response of the SSM, clearly indicating carryover effects due to both these parameters. While differences in intermeal duration between TM dinner and SSM breakfast (14.5 hour) during dinner intervention sessions and between TM breakfast and SSM lunch (3.5 hours) during breakfast intervention sessions may confound some of the differences observed, nonetheless this was the only study design that would have reflected a normal dietary pattern.

The increased PPG response after the SSM (breakfast) following high GI TM dinner compared with the low GI TM dinner may have arisen from the significantly greater baseline concentration of FFA concentration immediately prior to SSM (breakfast) following the high GI TM dinner intervention. This is similar to a previous observation that the fasting FFA concentration was positively correlated with glucose iAUC of a standardized breakfast. ${ }^{35}$ Furthermore, similar to our differences in fasted FFA concentration observation following an overnight fast, after high versus low GI dinner consumption the night before, an earlier study by Jenkins et al had also shown that consuming a bolus of $50 \mathrm{~g}$ glucose in comparison with continuous sipping over a 3-hour period led to greater FFA suppression over the postprandial period and significantly reduced blood glucose levels following a $5 \mathrm{~g}$ intravenous glucose tolerance test. ${ }^{36}$ Of course, it is well established that there is an inverse association between plasma FFA concentration and insulin sensitivity. ${ }^{37-40}$ Our study also showed greater suppression of FFA in the postprandial periods immediately following breakfast TM compared with the dinner TM. This, together with an increased glycemic response during the Di TM indicates reduced insulin sensitivity during dinner as compared with breakfast. Previous studies have indeed shown reduced insulin sensitivity during the evening as compared with the morning using insulin tolerance test or frequently sampled intravenous glucose tolerance test. ${ }^{412}$ Interestingly, insulin sensitivity in type 2 diabetics also changes with circadian rhythmicity, but seems to be lower in the morning than in the evening, possibly due to increased hepatic gluconeogenesis in type 2 diabetes, ${ }^{43} 44$ which suggests that our findings may not be entirely applicable to type 2 diabetics. Furthermore, the GI values of the TM reported in this study was estimated from the GI values of the same rice types from our previous studies rather than being directly measured in this study itself. Considering potential variations in GI of rice arising from subtle differences in growth conditions or milling, the actual GI of the TM may have been slightly different. As an additional limitation, it should be noted that due to the acute design of our study, the long-term validity of our findings would need to be further confirmed. Furthermore, we investigated only one specific time for breakfast and one specific time for dinner. Thus, our findings might have been somewhat variable for other timings of these meals. Nonetheless, by simultaneously measuring several markers at the same time, this study has produced a comprehensive dataset on the chronobiological impact of food consumption of variable GI on postprandial glucose homeostasis in a non-diabetic population.

In summary, our study specifically found that carbohydrate-rich meals taken at dinner gave rise to significantly adverse profile of glucose homeostasis as compared with the same meals taken at breakfast, independent of the GI content of the meal. These findings are likely to have public health implications in Asia where carbohydrate-rich, high GI meals are often consumed late during the day (often late evenings) due to cultural practices. For example, a recent study by Gupta $e t$ al in healthy adults in India found that $32 \%$ of daily intake of calories were consumed between $7.00 \mathrm{pm}$ and $11.00 \mathrm{pm}$, with the median dinnertime being after $10 \mathrm{pm} .{ }^{45}$ With the high prevalence of prediabetes worldwide, it will be advisable to particularly focus attention on the carbohydrate quality and quantity of the evening meals. The findings of our study also has significant implications on future design of epidemiological studies investigating GI of diet and disease risk associations. We recommend that the timing of intake of carbohydrates (ie, glycemic load) should also be assessed and reported as an additional variable. The results of this study prompt further research in the area of chrononutrition, particularly to investigate long-term effects of carbohydrate consumption at different times of the day.

Acknowledgements The authors would like to thank Shalini Ponnalagu, Siok Ching Chia, Bhupinder Kaur, Lijuan Sun, Joseph Lim, Susanna Lim, Cathy Mok, Tin Tin Aye, Corinne Ammon-Zufferey, Nathalie Frei, Patricia Leone, Fabienne Praplan and Andreas Rytz for their technical/regulatory assistance and the volunteers for taking part in this study.

Contributors SH, CJH, LE, CD, KM conceptualized and designed research. SH, MXNK and SLT were involved in the study conduct and data collection. CADC, LE and $\mathrm{SH}$ analyzed data or performed statistical analysis. SH and LE drafted the 
manuscript and all authors had been involved in the critical review of this work and approved the final manuscript. All authors agreed to be accountable for all aspects of the work. All authors affirm that authorship is merited based on the International Committee of Medical Journal Editors authorship criteria.

Funding This work was supported jointly by the Singapore Institute for Clinical Sciences, Agency for Science, Technology and Research (A*STAR), Singapore under the A*STAR BMRC SPF 2013/003 and Nestec RDSG015232.

Competing interests The authors have no competing interests to declare. LE, CADC, CD and KM are full-time employees of Nestlé Research.

\section{Patient consent for publication Not required.}

Ethics approval The study was approved by a Domain Specific Research Board (DSRB) ethics committee, Singapore (Reference: C/2016/00613) and was conducted in accordance with the Declaration of Helsinki 1983 and the Singapore Good Clinical Practice guidelines.

Provenance and peer review Not commissioned; externally peer reviewed.

Data availability statement De-identified participant data are available on reasonable request, within the regulatory framework of the authors' institutional policies, by emailing the corresponding author at 'jeya_henry@sics.a-star.edu.sg'.

Open access This is an open access article distributed in accordance with the Creative Commons Attribution Non Commercial (CC BY-NC 4.0) license, which permits others to distribute, remix, adapt, build upon this work non-commercially, and license their derivative works on different terms, provided the original work is properly cited, appropriate credit is given, any changes made indicated, and the use is non-commercial. See: http://creativecommons.org/licenses/by-nc/4.0/.

ORCID iD

Sumanto Haldar http://orcid.org/0000-0001-9559-5358

\section{REFERENCES}

1 Cavalot F, Pagliarino A, Valle M, et al. Postprandial blood glucose predicts cardiovascular events and all-cause mortality in type 2 diabetes in a 14-year follow-up: lessons from the San Luigi Gonzaga diabetes study. Diabetes Care 2011;34:2237-43.

2 Levitan EB, Song Y, Ford ES, et al. Is nondiabetic hyperglycemia a risk factor for cardiovascular disease? A meta-analysis of prospective studies. Arch Intern Med 2004;164:2147-55.

3 Blaak EE, Antoine J-M, Benton D, et al. Impact of postprandial glycaemia on health and prevention of disease. Obes Rev 2012:13:923-84.

4 Jenkins DJ, Wolever TM, Taylor RH, et al. Glycemic index of foods: a physiological basis for carbohydrate exchange. Am J Clin Nutr 1981:34:362-6.

5 Ludwig DS, Hu FB, Tappy L, et al. Dietary carbohydrates: role of quality and quantity in chronic disease. BMJ 2018;361:k2340.

6 Augustin LSA, Kendall CWC, Jenkins DJA, et al. Glycemic index, glycemic load and glycemic response: an international scientific consensus Summit from the International carbohydrate quality Consortium (ICQC). Nutr Metab Cardiovasc Dis 2015;25:795-815.

7 Barclay AW, Petocz P, McMillan-Price J, et al. Glycemic index, glycemic load, and chronic disease risk - a meta-analysis of observational studies. Am J Clin Nutr 2008;87:627-37.

8 Villegas R, Liu S, Gao Y-T, et al. Prospective study of dietary carbohydrates, glycemic index, glycemic load, and incidence of type 2 diabetes mellitus in middle-aged Chinese women. Arch Intern Med 2007:167:2310-6.

9 Finley CE, Barlow CE, Halton TL, et al. Glycemic index, glycemic load, and prevalence of the metabolic syndrome in the Cooper center longitudinal study. J Am Diet Assoc 2010;110:1820-9.

10 Vega-López S, Venn BJ, Slavin JL. Relevance of the glycemic index and glycemic load for body weight, diabetes, and cardiovascular disease. Nutrients 2018;10. doi:10.3390/nu10101361. [Epub ahead of print: 22 Sep 2018]

11 Schwingshackl L, Hoffmann G. Long-Term effects of low glycemic index/load vs. high glycemic index/load diets on parameters of obesity and obesity-associated risks: a systematic review and metaanalysis. Nutr Metab Cardiovasc Dis 2013;23:699-706.

12 Kristo AS, Matthan NR, Lichtenstein AH. Effect of diets differing in glycemic index and glycemic load on cardiovascular risk factors: review of randomized controlled-feeding trials. Nutrients 2013;5:1071-80.

13 Morris CJ, Yang JN, Garcia Jl, et al. Endogenous circadian system and circadian misalignment impact glucose tolerance via separate mechanisms in humans. Proc Natl Acad Sci U S A 2015;112:E2225-34.

14 Bass J, Takahashi JS. Circadian integration of metabolism and energetics. Science 2010;330:1349-54.

15 Van Cauter E, Polonsky KS, Scheen AJ. Roles of circadian rhythmicity and sleep in human glucose regulation. Endocr Rev 1997:18:716-38.

16 Dallmann R, Viola AU, Tarokh L, et al. The human circadian metabolome. Proc Natl Acad Sci U S A 2012;109:2625-9.

17 Panda S. Circadian physiology of metabolism. Science 2016;354:1008-15

18 Rudic RD, McNamara P, Curtis A-M, et al. Bmal1 and clock, two essential components of the circadian clock, are involved in glucose homeostasis. PLoS Biol 2004;2:e377.

19 Qin L-Q, Li J, Wang Y, et al. The effects of nocturnal life on endocrine circadian patterns in healthy adults. Life Sci 2003;73:2467-75.

20 Garaulet M, Madrid JA. Chronobiological aspects of nutrition, metabolic syndrome and obesity. Adv Drug Deliv Rev 2010;62:967-78

21 Asher G, Sassone-Corsi P. Time for food: the intimate interplay between nutrition, metabolism, and the circadian clock. Cell 2015;161:84-92.

22 Ribas-Latre A, Eckel-Mahan K. Interdependence of nutrient metabolism and the circadian clock system: importance for metabolic health. Mol Metab 2016:5:133-52.

23 Dickinson S, Colagiuri S, Faramus E, et al. Postprandial hyperglycemia and insulin sensitivity differ among lean young adults of different ethnicities. J Nutr 2002;132:2574-9.

24 Lee JWR, Brancati FL, Yeh H-C. Trends in the prevalence of type 2 diabetes in Asians versus whites: results from the United States National health interview survey, 1997-2008. Diabetes Care 2011;34:353-7.

$25 \mathrm{Tan} \mathrm{VMH}$, Wu T, Henry CJ, et al. Glycaemic and insulin responses, glycaemic index and insulinaemic index values of rice between three Asian ethnic groups. Br J Nutr 2015;113:1228-36.

26 Ranawana DV, Henry CJK, Lightowler HJ, et al. Glycaemic index of some commercially available rice and rice products in Great Britain. Int J Food Sci Nutr 2009;60 Suppl 4:99-110.

27 Kaur B, Ranawana V, Teh A-L, et al. The impact of a low glycemic index (Gi) breakfast and snack on daily blood glucose profiles and food intake in young Chinese adult males. $J$ Clin Transl Endocrinol 2015;2:92-8

28 Leung GKW, Huggins CE, Bonham MP. Effect of meal timing on postprandial glucose responses to a low glycemic index meal: a crossover trial in healthy volunteers. Clin Nutr 2019;38:465471:465-71.

29 Gibbs M, Harrington D, Starkey S, et al. Diurnal postprandial responses to low and high glycaemic index mixed meals. Clin Nutr 2014:33:889-94.

30 Melanson KJ, Greenberg AS, Ludwig DS, et al. Blood glucose and hormonal responses to small and large meals in healthy young and older women. J Gerontol A Biol Sci Med Sci 1998:53:B299-305.

31 Ma RCW, Chan JCN. Type 2 diabetes in East Asians: similarities and differences with populations in Europe and the United States. Ann N Y Acad Sci 2013;1281:64-91.

32 Wolever TM, Jenkins DJ, Ocana AM, et al. Second-meal effect: lowglycemic-index foods eaten at dinner improve subsequent breakfast glycemic response. Am J Clin Nutr 1988;48:1041-7.

33 Lee S-H, Tura A, Mari A, et al. Potentiation of the early-phase insulin response by a prior meal contributes to the second-meal phenomenon in type 2 diabetes. Am J Physiol Endocrinol Metab 2011;301:E984-90.

34 Nilsson A, Granfeldt Y, Ostman E, et al. Effects of Gi and content of indigestible carbohydrates of cereal-based evening meals on glucose tolerance at a subsequent standardised breakfast. Eur $J$ Clin Nutr 2006;60:1092-9.

35 Nilsson A, Ostman E, Preston T, et al. Effects of Gi vs content of cereal fibre of the evening meal on glucose tolerance at a subsequent standardized breakfast. Eur J Clin Nutr 2008:62:712-20.

36 Jenkins DJ, Wolever TM, Ocana AM, et al. Metabolic effects of reducing rate of glucose ingestion by single bolus versus continuous sipping. Diabetes 1990;39:775-81.

37 Griffin ME, Marcucci MJ, Cline GW, et al. Free fatty acid-induced insulin resistance is associated with activation of protein kinase $\mathrm{C}$ theta and alterations in the insulin signaling cascade. Diabetes 1999;48:1270-4

38 Frohnert $\mathrm{Bl}$, Jacobs DR, Steinberger J, et al. Relation between serum free fatty acids and adiposity, insulin resistance, and cardiovascular risk factors from adolescence to adulthood. Diabetes 2013:62:3163-9. 
39 Johns I, Goff L, Bluck LJ, et al. Plasma free fatty acids do not provide the link between obesity and insulin resistance or $\beta$-cell dysfunction: results of the reading, imperial, Surrey, Cambridge, kings (RISCK) study. Diabet Med 2014;31:1310-5.

40 Boden $\mathrm{G}$. Free fatty acids, insulin resistance, and type 2 diabetes mellitus. Proc Assoc Am Physicians 1999;111:241-8.

41 Morgan LM, Aspostolakou F, Wright J, et al. Diurnal variations in peripheral insulin resistance and plasma non-esterified fatty acid concentrations: a possible link? Ann Clin Biochem 1999;36:447-50.

42 Lee A, Ader M, Bray GA, et al. Diurnal variation in glucose tolerance. cyclic suppression of insulin action and insulin secretion in normalweight, but not obese, subjects. Diabetes 1992;41:750-9.
43 Boden G, Chen X, Urbain JL. Evidence for a circadian rhythm of insulin sensitivity in patients with NIDDM caused by cyclic changes in hepatic glucose production. Diabetes 1996;45:1044-50.

44 Chang CR, Francois ME, Little JP. Restricting carbohydrates at breakfast is sufficient to reduce 24-hour exposure to postprandial hyperglycemia and improve glycemic variability. Am J Clin Nutr 2019;109:1302-9.

45 Gupta NJ, Kumar V, Panda S. A camera-phone based study reveals erratic eating pattern and disrupted daily eating-fasting cycle among adults in India. PLoS One 2017;12:e0172852. 